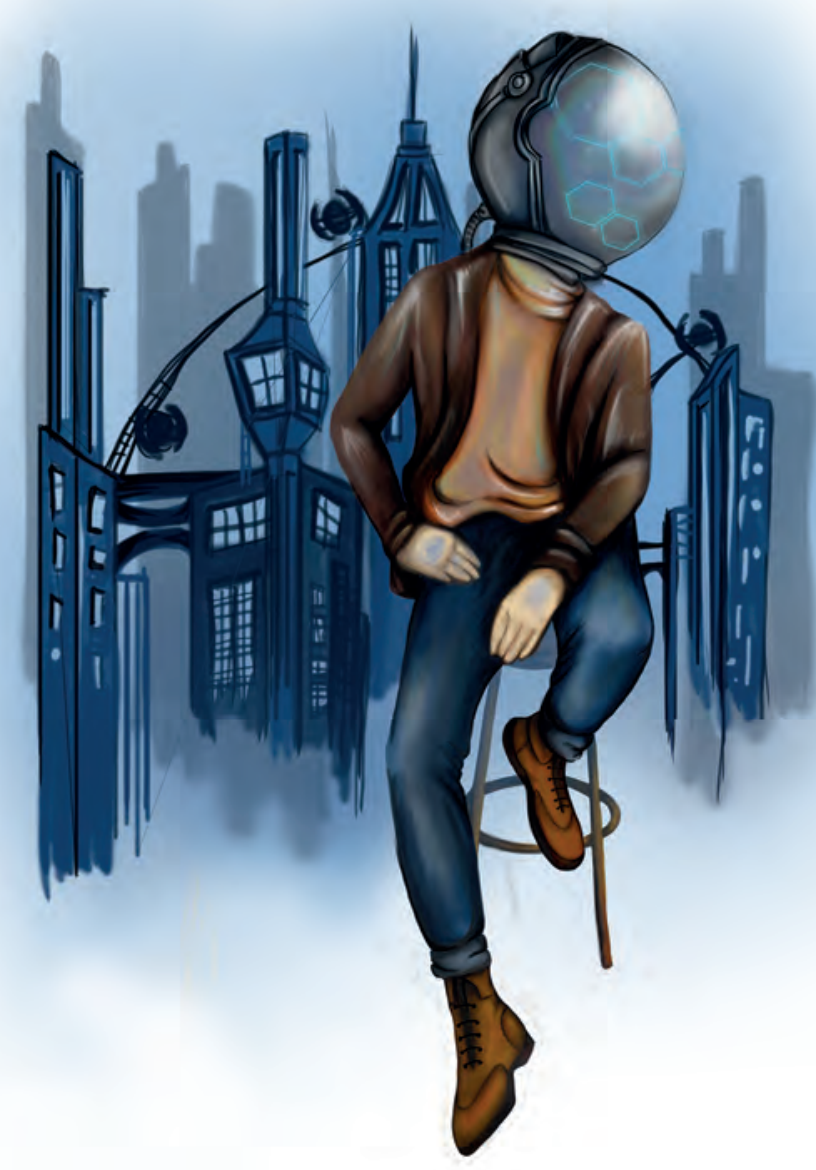

- Capítulo 2 -

\title{
Simulador de análisis prospectivo
}

\section{Autores}

Ronald Mauricio Martínez Contreras. Administrador de empresas egresado del Politécnico Grancolombiano y Magister en Administración Financiera de la universidad Sergio Arboleda, con más de 10 años de experiencia en cargos directivos y ejecutivos en empresas del sector real, financiero y de servicios, 
vinculado al sector educativo desde hace más de cuatro años y en la actualidad es Director del Departamento Académico de Administración del Politécnico Grancolombiano.

Correspondencia: romartin@poligran.edu.co

Luis Martín Trujillo Flórez. Ingeniero electricista egresado de la Universidad Nacional de Colombia. Máster en Edición Universitaria Universidad de Salamanca. Maestría en Ambientes Virtuales de Aprendizaje Universidad de Panamá. Especialista en virtualidad Centro de Altos Estudios de Argentina. Coordinador del Laboratorio de innovación pedagógica Politécnico Grancolombiano.

Correspondencia:mtrujilo@poligran.edu.co

\section{Diseñador de interfaces del simulador}

Mónica Carrero Becerra. Diseñadora gráfica e ilustradora del Politécnico Grancolombiano. Tecnóloga en multimedia del Servicio Nacional de Aprendizaje. Actualmente se desempeña como diseñadora del Laboratorio de innovación pedagógica del Politécnico Grancolombiano.

Correspondencia:mncarrero@poligran.edu.co

\section{Resumen}

Ante la necesidad de contar con un software de prospectiva que integrara diferentes técnicas y herramientas de análisis y a su vez, tuviera un alto componente pedagógico, se desarrolló el proyecto denominado "Simulador de Análisis Prospectivo". Con este propósito se hizo una revisión conceptual y metodológica del proceso de análisis prospectivo con el fin de desarrollar la herramienta tecnología más idónea para la identificación de escenarios futuros partiendo del análisis de las tendencias clave de índole interno y externo que pueden afectar a una organización en un horizonte temporal de diez años. Gracias a este proceso investigativo se desarrolló una herramienta tecnológica que facilita el proceso de aprendizaje de los estudiantes con una metodología teórico-práctica y que traerá beneficios académicos en dos frentes, en primer 
lugar, mejorará la calidad de los proyectos de investigación de los estudiantes $y$, en segundo lugar, fortalecerá la relación con el sector externo debido al mejoramiento de la calidad en el análisis prospectivo realizado por los estudiantes.

\section{Palabras claves}

Análisis Estructural, DOFA, Escenarios, Futuribles, Hipótesis, Mic-Mac, PESTEL, Prospectiva.

\section{Introducción}

El análisis prospectivo es uno de los conceptos administrativos que más debate ha generado en las últimas décadas debido a que la posibilidad de idear escenarios futuros para algunos expertos todavía se acerca más al campo de la adivinación que al de la administración. Sin embargo, los cambios abruptos en los mercados que provocaron los cambios en las dinámicas empresariales, además de las crisis financieras de nuestro siglo, le han dado mayor relevancia a la creación de escenarios futuros que conduzcan a una planeación estratégica. De hecho, recientemente nació un concepto nuevo denominado Riesgo Estratégico en el que se enmarca la planeación estratégica y la prospectiva empresarial (Ryan, 2017), bajo esta óptica la planeación por escenarios se vuelve una necesidad de todas las empresas porque les permite anticiparse a los riesgos potenciales que puedan enfrentar en el corto, mediano y largo plazo.

El propósito del análisis prospectivo es la identificación de diferentes escenarios posibles a partir del análisis de las tendencias clave que pueden afectar el desempeño futuro de una organización. Este análisis y depuración de variables se hace mediante diferentes técnicas y herramientas que ayudan a que el resultado sea el más acertado posible. En este mismo contexto diversos autores, a lo largo de la historia, han desarrollado variadas herramientas como el método Delphi, las matrices de análisis estructural, el método de escenarios y los arboles de competencias que han dotado al análisis prospectivo bases metodológicas que sustentan su aplicabilidad en la gestión empresarial.

A partir de tales herramientas y ante la necesidad de fortalecer en los estudiantes la capacidad de realizar un adecuado análisis estratégico surge la oportunidad 
de desarrollar una estrategia de aprendizaje que le permita al aprendiz integrar diferentes técnicas de análisis prospectivo, de tal manera que utilizando un método organizado pueda analizar y depurar los datos hasta llegar a los escenarios futuros más probables a los que se puede enfrentar una compañía en un horizonte de diez años. De esta manera, disminuir considerablemente la incertidumbre asociada a los sucesos futuros y realizar una planeación más estructurada.

El inicio del proceso se inició con la creación de un método para el análisis prospectivo que reúne varias técnicas combinadas con aportes del autor, dicho método fue implementado en un archivo de Excel que los estudiantes pueden trabajar. Sin embargo, para lograr una experiencia más amplia de aprendizaje, se desarrolló un simulador que integra el método elaborado (incluye un análisis de probabilidades que no está en el Excel) con una estrategia pedagógica que involucra la simulación al módulo o asignatura de Pensamiento Estratégico y Prospectiva.

Desde lo académico, el simulador le permite al estudiante hacer un diagnóstico de los escenarios futuros para cualquier empresa real, a través de un proceso metódico que lo lleva a la selección de las tendencias clave hasta llegar a la construcción de los escenarios más probables y finalmente a la selección del escenario meta.

En el desarrollo del simulador se integraron en un sistema dos funciones inexistentes en el mercado; la primera, integrar en un sólo software diversas técnicas de análisis prospectivo; la segunda, está enfocada en brindar una solución pedagógica amigable para los estudiantes de los programas de pregrado y posgrado de Administración de Empresas del Politécnico Grancolombiano.

\section{Marco teórico}

\section{Problemática que atiende}

En el aprendizaje de la prospectiva existe una dificultad que se evidencia en el desarrollo de los proyectos de aula y se vuelve la pregunta inicial para este proyecto: ¿Cómo evidenciar en un contexto real los conceptos de prospectiva que estudia el aprendiz? 
Esa es una de las mayores dificultades que se encontró en el desempeño de los estudiantes. Gran parte de lo que ve el estudiante se queda en conceptos y definiciones, poco se logra en la construcción de escenarios. De allí, surge la necesidad de crear una estrategia de aprendizaje que permita al estudiante aplicar lo visto en la teoría del módulo o asignatura a una empresa real y lo lleve a establecer un escenario - meta que le permita diseñar su estrategia gerencial.

En el módulo o asignatura de Pensamiento Estratégico y Prospectiva (en donde se aplica esta experiencia) el proyecto de aula consiste en tomar una organización y realizar un análisis prospectivo de la misma, con dicho análisis se tiene el insumo para elaborar la planeación estratégica de la empresa con miras a ser aplicada en el futuro y que le produzca mejores resultados. Con este proyecto de aula se evidencian algunas dificultades por parte de los educandos, el primero de ellos es la adecuada definición de variables que conduzcan a un correcto análisis, para ello el tutor los orienta con el fin de que ellos puedan depurar sus variables y hacer un análisis más cercano a la realidad empresarial. La segunda dificultad es que los estudiantes en su gran mayoría no saben aplicar los métodos de análisis dedicándose más a la parte procedimental que a la parte de diagnóstico, definición de variables y análisis que es lo más importante, por consiguiente, pierden mucho tiempo en la aplicación de los métodos y al no obtener unas hipótesis adecuadas los escenarios no llegan a ser del todo consistentes, por ende, la planeación estratégica con el escenario meta no logra lo que se espera.

A partir de esta dificultad nace la primera problemática a la que se enfrentó el proyecto, integrar un método de análisis prospectivo que iniciara con la identificación de las variables, su definición, su nivel de impacto, hasta llegar a la selección del escenario meta. Para esto fueron consultadas e integradas varias metodologías ya existentes, lo cual derivó en la construcción de un método con aportes propios del autor.

El método inicia con la definición de las variables que pueden afectar en el futuro a la empresa a nivel interno y externo, también se tiene en cuenta la evaluación del posible impacto en la compañía. En cada análisis se depuran las variables hasta cuantificar posibles probabilidades de ocurrencia, asimismo se analiza la dependencia que tienen con otras variables y cómo estas afectan a la compañía directa e indirectamente. De esa manera, la construcción de escenarios se vuelve un juego que depura las rutas de mayor probabilidad para construir desde allí los escenarios más factibles. Al final del proceso se 
encuentran los diez escenarios con una alta probabilidad de ocurrencia para que el estudiante elija el escenario meta y desde allí construya su estrategia gerencial. Es decir, el proceso involucra lo que un gerente debe hacer cuando aplica la prospectiva a su organización. Luego le apuesta a un escenario meta donde enfoca su estrategia gerencial que es la parte final de la experiencia de aprendizaje.

Con este método de análisis se procedió a crear un aplicativo en Excel que integra las técnicas y el estudiante ingresa la información permitiéndole superar la dificultad procedimental, de esta manera, puede centrarse más en el análisis prospectivo. El aplicativo acompaña al estudiante hasta la creación de las diferentes hipótesis que afectarán los escenarios.

A partir del aplicativo en Excel se procedió a desarrollar el simulador que incluye la evaluación de las hipótesis de acuerdo con su probabilidad de ocurrencia, dentro de la programación se tuvo la gran dificultad de hacer un algoritmo que analizara las rutas de mayor probabilidad y fuera construyendo los escenarios más probables. Para superar este obstáculo se recurrió a la programación de grafos y árboles de decisiones, al final el software le entrega al estudiante los diez escenarios más probables.

Para este aspecto se estudiaron otras experiencias similares y se encontró que el simulador desarrollado en este proyecto supera las iniciativas anteriores, como las desarrolladas por el Ministerio de Relaciones Exteriores de Francia junto con France Telecom y otras empresas asociadas quienes han creado herramientas gratuitas de análisis prospectivo.

Los dos elementos que se trabajan con el simulador son la incorporación de todo el proceso de análisis prospectivo en un solo software y una plataforma amigable con el usuario que le permita tener claridad de los resultados encontrados en todas las etapas del análisis prospectivo. Además, el simulador permite que los contenidos temáticos del módulo relacionado sean trabajados de forma práctica, de tal manera que los temas pueden ser contrastados con la realidad empresarial. Tal vez la característica más importante que tiene el simulador es que puede ser aplicado a cualquier tipo de empresa y en cualquier etapa de su ciclo de vida. 


\section{Estrategia de aprendizaje}

El aprendizaje de la prospectiva en la era del conocimiento cada día adquiere mayor relevancia, hoy en día el análisis de lo que puede suceder en el futuro no solo activa la imaginación sino la creatividad a través de la generación de ideas e hipótesis que permitan adelantarse a lo que pueda suceder, también permite desarrollar la capacidad de innovar al implementar en la estrategia gerencial procesos y proyectos que no se han realizado, es tener la capacidad de afrontar nuevos retos. Ya no se trate de dejar el futuro al azar sino analizar las probabilidades de acuerdo con los comportamientos, la información de lo que ocurre en el entorno, las dinámicas económicas y sociales que permitan a futuro tomar las decisiones más acertadas que permitan hacer de la incertidumbre una oportunidad.

Según Quiroga (2008) "La gestión del conocimiento y el uso de herramientas que faciliten el diseño y construcción del futuro de las empresas, disminuyen los riesgos organizacionales". Significa que las decisiones empresariales tomadas en ambientes de incertidumbre conllevan a altos riesgos debido a la competencia global. Es por lo que la creación de escenarios que permitan la adecuada toma de decisiones por parte de los actores directamente relacionados disminuye estos riesgos en forma considerable, percibiéndose la importancia y necesidad que la empresa posea herramientas de gestión como la prospectiva empresarial, que permite diseñar y construir el futuro con el menor riesgo posible y con el mayor grado de probabilidad de ocurrencia, aún en ambientes de incertidumbre (Quiroga, 2008).

La disminución de la incertidumbre fue el punto de partida para la estrategia pedagógica que después dio origen al simulador. La incertidumbre disminuye si identificamos las mayores probabilidades de ocurrencia de un fenómeno. Por tal razón, tanto el simulador como la experiencia de aprendizaje se fundamentan en los principios básicos de la prospectiva que son:

- No existe un solo camino para el negocio.

- El pasado no explica el futuro.

- Se requiere una gestión de los negocios activa y creativa.

Por lo tanto, atendiendo el primer principio, el proceso de simulación se basa en que el estudiante seleccione las posibles variables y las clasifique de acuerdo con los factores externos e internos que puedan tener incidencias futuras en la organización. 
Para el segundo principio, a partir de las tendencias claves del negocio se plantean hipótesis de las cuales se crean los escenarios futuros.

Para el tercer principio, la propuesta es que el estudiante, a partir de su experiencia de simulación y con su escenario meta, construya la estrategia gerencial completa para implementar en la compañía.

Teniendo estos tres principios empezamos a trabajar en una estrategia pedagógica que nos permitiera evidenciar un análisis prospectivo en una empresa real. Como primera medida se implementó en el módulo o asignatura un proyecto de aula donde los equipos de estudiantes seleccionaban una empresa y aplicaban algunos métodos de análisis como el Delphi, el análisis PESTEL, el método MIC-MAC, entre otros. En la mayoría de casos el estudiante se complicaba más en la aplicación de los métodos que en procurar construir una información de calidad que permitiera un mejor diagnóstico de la empresa, pues la mayor dificultad radicaba en una correcta definición de las variables que afectan la empresa y de las hipótesis para la creación de escenarios.

Pensamos, entonces, en una estrategia que hiciera que los estudiantes se preocuparán más por el análisis que por la aplicación de un método específico. Esa es una de las ventajas del simulador, que permite al estudiante concentrarse en un análisis más profundo de la empresa que en seguir los pasos adecuados para aplicar el método. Para ello se creó un aplicativo en Excel que se aplicó en los estudiantes con buenos resultados. De dicho aplicativo nace la idea de convertirlo en un simulador, pues se pretende un aprendizaje más significativo para el estudiante, y no sólo se enfrenta al análisis, sino que existe un gerente que emula un escenario empresarial y le hace solicitudes al estudiante, pasando de la mera aplicación del método que ofrece el Excel a una experiencia de un ambiente virtual de aprendizaje.

La parte teórica es trabajada con los materiales del módulo de Pensamiento Estratégico y Prospectiva. (Lecturas fundamentales, objetos virtuales de aprendizaje, teleconferencias, encuentros sincrónicos, etc.). El área de la aplicación o la práctica se compone de dos partes, la primera, es el análisis prospectivo de la empresa que se trabaja en el simulador, el cual da como resultado la elección del escenario meta. La segunda, es tomar ese escenario meta y de manera colaborativa deben construir la planeación estratégica de la empresa. 
Para ello, se recurre a la aplicación de métodos activos de aprendizaje, donde la primicia es aprender haciendo, en este caso en particular, el propósito es que el estudiante aplique de forma práctica en un contexto real lo que ve de manera teórica.

La base pedagógica de la experiencia es el aprendizaje vivencial cuyo fundamento consiste en que los estudiantes aprenden mejor cuando participan activamente en experiencias abiertas de aprendizaje que cuando lo hacen de manera pasiva, en actividades estructuradas como sucedía en el módulo. Por lo tanto, el Aprendizaje Vivencial permite que el estudiante aplique lo aprendido en situaciones reales. De esa manera, el estudiante se enfrenta a problemas, plantea por sí mismo diferentes hipótesis de solución, prueba las soluciones e interactúa con sus compañeros en un contexto determinado (Moore, 2013).

La metodología que se deriva del aprendizaje vivencial y se aplica a los estudiantes es el aprendizaje basado en proyectos donde los estudiantes deben generar un producto, presentación, o ejecución de la solución (Larmer, 2015). Los estudiantes inician con la selección de una empresa del sector real, después establecen las variables que van a analizar, realizan el análisis prospectivo para obtener el escenario meta y de manera colaborativa desarrollan la planeación estratégica que es el producto con el que finaliza la experiencia.

El Aprendizaje Vivencial es un enfoque holístico integrador del aprendizaje, que combina la experiencia, la cognición y el comportamiento (Akella, 2010). El simulador sirve para que los estudiantes obtengan el análisis prospectivo, pero no pueden llegar a este si no tienen claro el comportamiento y los conceptos para concluir en dicho análisis. Con el fin de integrar la experiencia y comprometer a cada estudiante se plantea una simulación individual, la cual se hace con la empresa que seleccionó el equipo y posteriormente cada miembro del equipo propone sus variables y simula por separado. Superada esta etapa, se realiza una reunión de equipo para consolidar la información y hacer una simulación grupal. A medida que hacen las simulaciones envían los resultados al tutor para obtener la retroalimentación, si es correcta el tutor da su aprobación para que continúen, si deben hacer cambios deben retomar y realizar los ajustes. Cuando los estudiantes terminan y obtienen los diez escenarios, de manera conjunta deben seleccionar un escenario meta. A partir de ese escenario meta los estudiantes deben construir de manera colaborativa la estrategia empresarial y el proceso cognitivo se fortalece porque evidencia la aplicación de la teoría en una empresa real. 
La figura 1. Muestra las etapas de la estrategia pedagógica para la simulación.

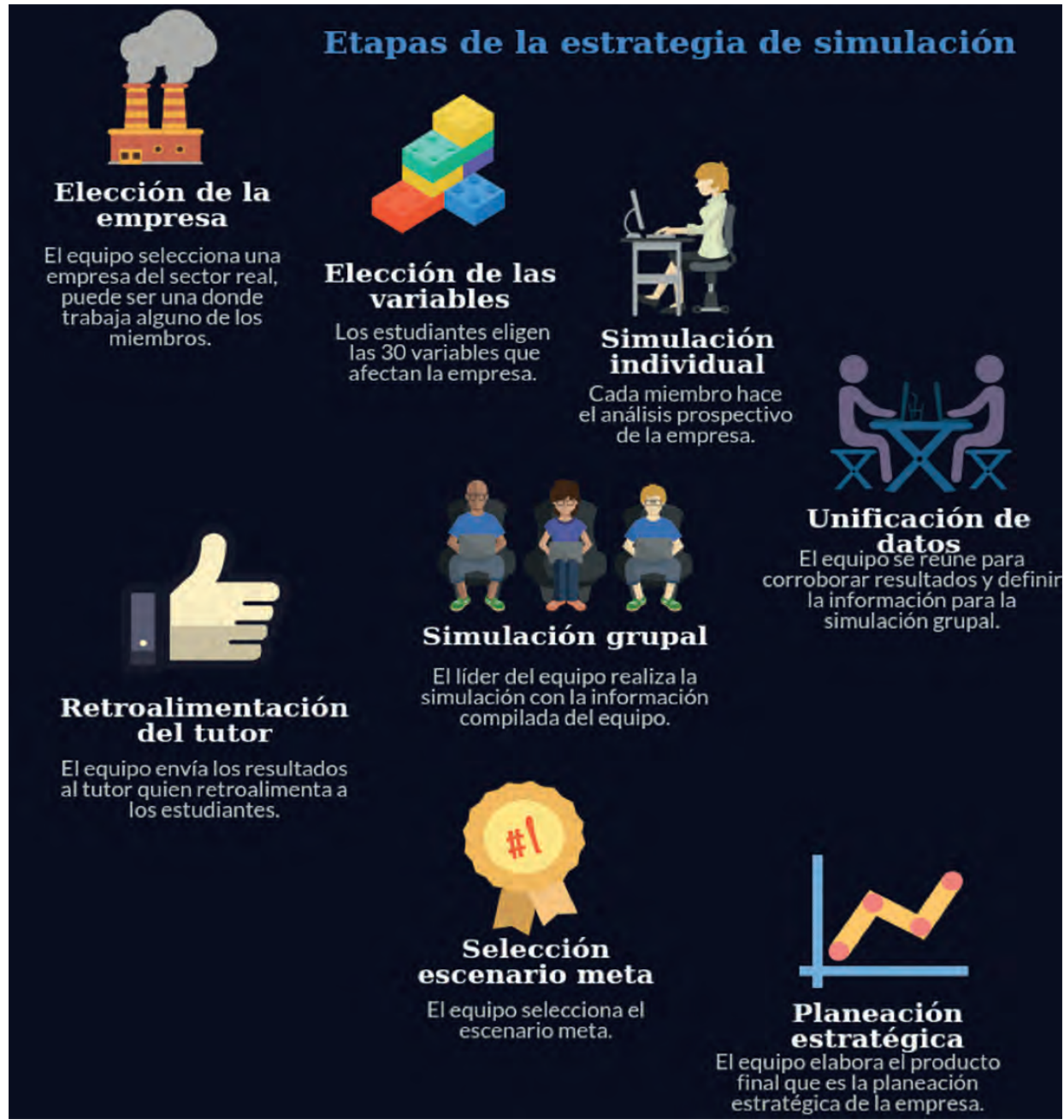

Figura 1. Etapas de la estrategia pedagógica de simulación.

Fuente: Elaboración propia (2017).

Como producto de la experiencia de aprendizaje los estudiantes entregan un documento que contiene el análisis prospectivo que la descargan de los resultados del simulador y la estrategia empresarial.

Los estudiantes trabajan el proyecto asignado con la empresa del sector real, que ellos eligieron, de manera que el resultado final es un proyecto para su aprendizaje (Moursund, 1999) y para su vida, porque si hacen trabajo con una empresa en la cual trabajan ofrecen soluciones a contextos reales. 
La motivación intrínseca parte del propio sujeto, de su interés y disposición hacia el disfrute de algo (Aguado, 2005). Para la experiencia se incluye un componente que favorece el aprendizaje motivacional y aumenta el interés del estudiante, este elemento se propone desde el reto, que se gesta a través de un personaje que es un gerente el cual le va indicando los retos que debe realizar el estudiante como si estuviera en una empresa real.

Asimismo, se creó un personaje guía que acompaña al estudiante durante el proceso de simulación. Este personaje se llama Mila y su objetivo es orientar al aprendiz en cada uno de los pasos que debe realizar en la simulación, en algunos casos brinda explicaciones cortas de la teoría y en otros momentos imparte instrucciones de las tareas que debe realizar el estudiante. Este personaje es transversal a varios proyectos de simulación de la institución.

La experiencia de simulación además cuenta con una guía metodológica que orienta las competencias a desarrollar en todo el proyecto, le explica a los estudiantes cómo será su proceso de simulación y les da indicaciones para los entregables, asimismo, los criterios de evaluación que se tienen para toda la propuesta.

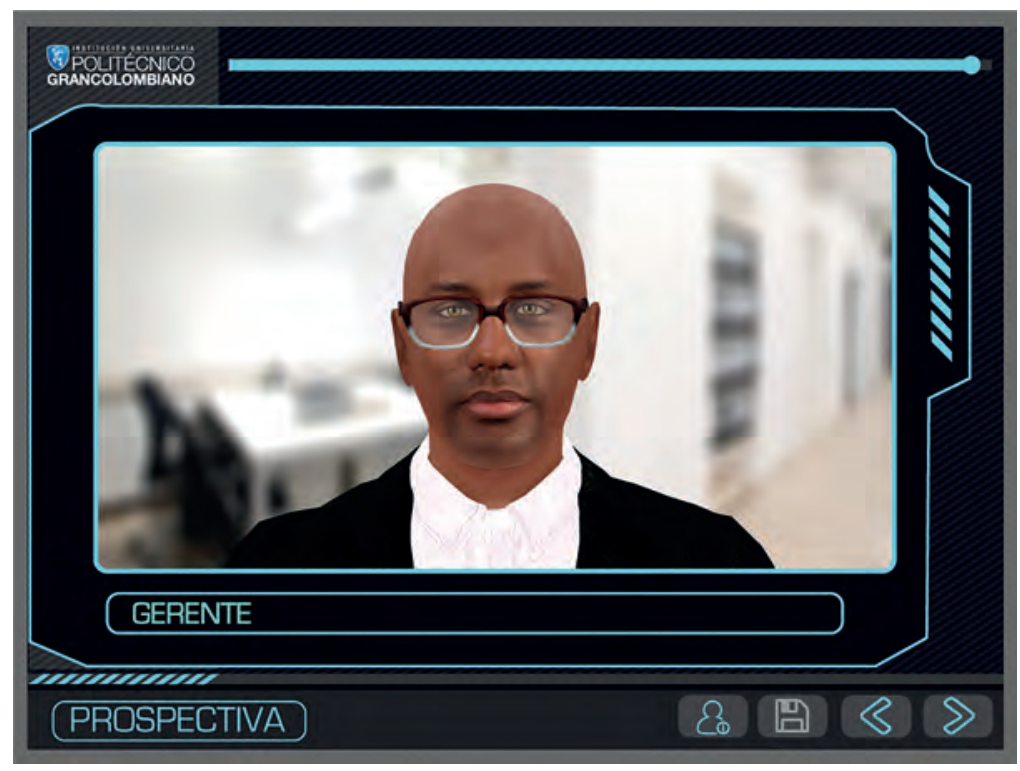

Figura 2. Gerente general de la empresa para la simulación.

Fuente: Politécnico Grancolombiano. 


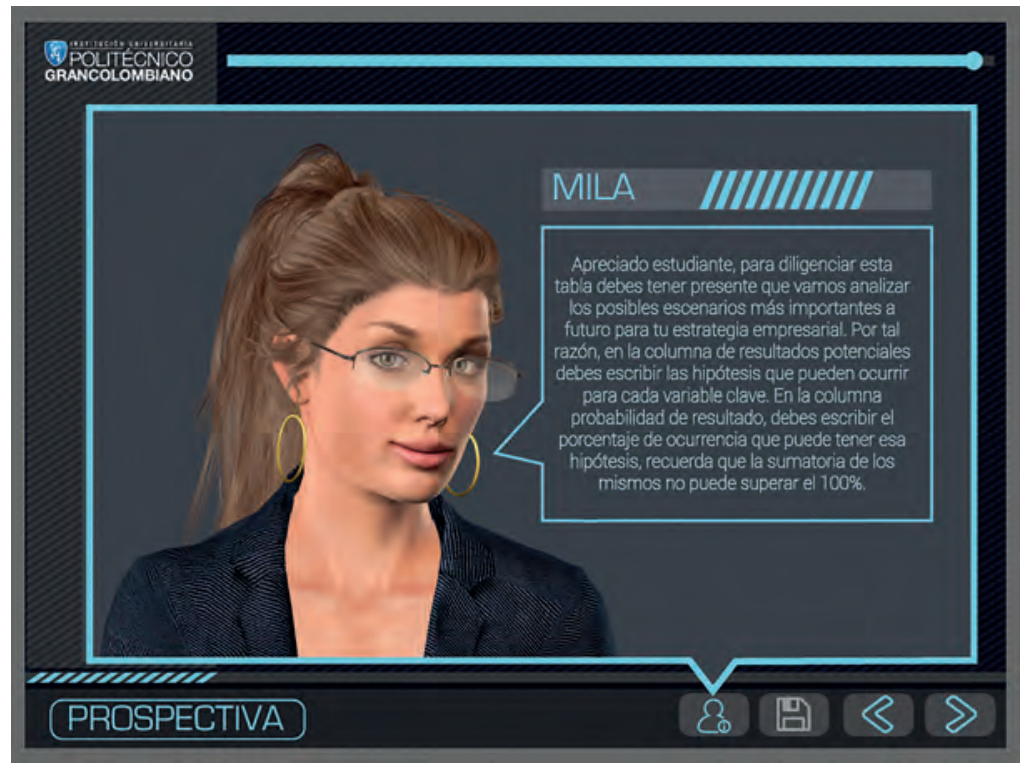

Figura 3. Personaje de ayuda al estudiante.

Fuente: Politécnico Grancolombiano.

Por otro lado, para la simulación es fundamental el trabajo colaborativo y los compromisos del estudiante con su equipo, pues una de las situaciones que se han evidenciado son las malas prácticas que tienen los estudiantes para trabajar colaborativamente, entre ellas: la preferencia por trabajar de manera individual para lo cual se dividen los trabajos para que cada miembro del grupo realice el proyecto de aula de un módulo, de esa manera, uno aprende y los demás quedan inscritos en el proyecto. Otra mala práctica es que solo trabaja uno del grupo y los otros solo escriben el nombre. Otra es que algunos estudiantes nunca se hacen presentes y sus compañeros deben asumir el trabajo de todo el equipo. En este caso el simulador está pensado para que todos trabajen porque la simulación grupal se debe hacer con el compilado hecho en la simulación individual. Si el estudiante no simula individualmente ninguno de los miembros del grupo puede acceder a la del grupo, esto ejerce una presión social para que todos se comprometan con el equipo. También existe la figura del líder, quien digita los datos del grupo para la simulación, por ende, si el grupo quiere un líder eficiente debe responder con el equipo, y si el líder es eficiente el trabajo de todo el equipo fluye.

Para lograr una transparencia el simulador elige aleatoriamente los miembros del grupo y el líder de este, esto se hace por varias razones, la primera de ellas es que no existan grupos incompletos y que no quede todo el proceso como 
única responsabilidad del líder y que los demás no se comprometan con el trabajo. De igual manera, el hecho de que sean aleatorios se hace con el sentido de emular lo que pasa en un ambiente laboral, donde el individuo debe estar en capacidad de trabajar con cualquier persona sin prejuicio alguno. Esta práctica hace parte de la implementación inicial del módulo y cuando llegan a la parte colaborativa ya están comprometidos con el equipo y se deben producir los resultados esperados.

\section{Modelo para la simulación}

El origen del análisis prospectivo se remonta al año 1945 cuando el Departamento de Defensa de los Estados Unidos crea el proyecto Rand CO. mediante el cual se da origen al laboratorio de ideas y se desarrollan las primeras técnicas de análisis del futuro como el método Delphi, Dalkey y Olaf (1962) y el análisis morfológico descrito por Baena (2009). Sin embargo, es en Francia donde se desarrolla y estructura el concepto de análisis prospectivo que hoy conocemos. La denominada escuela francesa nació con el aporte de Bertrand de Jouvenel, en Mojica (2006), en donde manifiesta que existen muchos futuros posibles denominados futuribles. Una década después esta escuela se fortalece con las ideas de Godet (2000) que unifica los conceptos de prospectiva con la planeación estratégica, tal vez su mayor aporte radique en la unificación de herramientas técnicas para el análisis con el propósito de dar un sustento metodológico al análisis prospectivo.

Mojica (1992) define la prospectiva como "La identificación de un futuro probable y deseable, que depende del conocimiento que tenemos, sobre las acciones que el hombre quiera emprender; $y$ son los actores quienes deciden qué se debe conservar y qué se debe cambiar".

Al partir del hecho de la existencia de diferentes futuros para un negocio es necesario identificarlos y tenerlos en cuenta:

- Futuro posible: Es todo aquello que puede suceder en el futuro

- Futuro probable: Son los futuros que pueden suceder de acuerdo con la aplicación de métodos estadísticos que brindan cierto nivel de confianza.

- Futuro deseable: Es el futuro que más se ajusta a las necesidades de la compañía con base en los futuros probables.

- Futuro factible: Finalmente, el futuro factible, es aquel que es posible, 
probable y deseable y se puede llevar a cabo. Este es el denominado escenario meta en el cual la compañía debe direccionar su planeación estratégica.

Sin embargo, para llegar al escenario meta es necesario valerse de diferentes herramientas de análisis estratégico que permitan identificar las tendencias clave del negocio. La herramienta por excelencia para la identificación de tendencias es el análisis PESTEL; este análisis aplicado a la prospectiva se encarga de identificar los posibles fenómenos políticos, económicos, sociales, tecnológicos, ecológicos y legales a los que la empresa tendrá que hacerle frente en un horizonte de diez o quince años. Como complemento al análisis externo, la compañía también debe analizar cómo se encontrarán sus posibles aspectos internos en el futuro.

Para el desarrollo de estos análisis es importante apoyarse en herramientas como el método Delphi o panel de expertos, con el fin de que la identificación de tendencias sea lo más objetiva posible.

Luego de identificar las tendencias clave pare el negocio se deben clasificar, con este propósito se desarrollaron las matrices de análisis estructural de las cuales nos enfocamos en la matriz de impactos cruzados y en el análisis MICMAC.

La matriz de impactos cruzados permite cuantificar las tendencias de acuerdo con su nivel de dependencia e influencia con respecto a las demás variables. La dependencia se refiere a que tanto depende el resultado de la variable analizada de los resultados que se den cada una de las tendencias clave del negocio (estas se califican en una escala de cero a tres donde cero es una dependencia nula y tres es una dependencia alta). Mientras que las influencias evalúan qué tanto influye la variable a analizar en el resultado de las demás tendencias clave del negocio. Al igual que con las dependencias estas se califican en una escala de cero a tres donde cero es una influencia nula y tres es una influencia alta (Arango y Cuevas, 2014).

De la sumatoria de los valores numéricos obtenidos en la matriz de impactos cruzados, se obtienen los puntos del grafico para el análisis MIC MAC. Este gráfico tiene cuatro cuadrantes o zonas que son: 
- Zona de poder: en esta zona se encuentran las tendencias sobre las cuales se debe desarrollar la estrategia de la compañía, ya que en esta se ubican las tendencias que tienen mucha influencia en el resultado de las demás y en contraste su resultado depende poco de otras variables.

- Zona de conflicto: en esta zona se encuentran las tendencias de riesgo para la compañía, son variables que tienen una influencia importante sobre el resultado de otras, pero su resultado depende de variables que no son del control de la compañía, por tal motivo, estas variables se deben gestionar para mitigar los riesgos de sus resultados potenciales.

- Zona de resultado: en esta zona se ubican las tendencias que tienen poca influencia en las demás, pero que a su vez su resultado depende en gran medida de otras variables, estas son tendencias que potencialmente podrían pasar a la zona de conflicto convirtiéndose en variables de riesgo para la compañía.

- Zona de autonomía: en esta zona se ubican las tendencias que tienen el menor impacto en el futuro de la compañía. Ya que sus niveles de influencia y dependencia son bajos.

De acuerdo con estas zonas del análisis MIC-MAC se identifican las tendencias clave para la estrategia ubicadas en la zona de poder y las tendencias de riesgo ubicadas en la zona de conflicto. Para cada una de ellas se deben desarrollar las hipótesis o posibles resultados potenciales que podría tener cada una. Para esta etapa es importante recurrir de nuevo al método Delphi con el objetivo de lograr resultados más precisos.

Para el desarrollo del simulador se empleó la lógica utilizada en mercados de predicciones mediante la cual para cada variable existe un número determinado de resultados potenciales, en nuestro caso tres, los cuales deben ser exhaustivos y excluyentes; esto quiere decir que los resultados deben cubrir el total de las probabilidades para sumar un total del $100 \%$ y por otro lado, excluyente se refiere a que si se diera la hipótesis $A$, como consecuencia no se puede dar la hipótesis B ni la hipótesis C, (Tziralis y Tatsiopoulos, 2012). Bajo esta premisa se desarrolla la matriz de hipótesis del simulador.

Finalmente, a partir de las hipótesis propuestas se identifican los diez escenarios más probables mediante la combinación de todas las hipótesis potenciales que son susceptibles de tener ocurrencia simultánea, la construcción de los escenarios se da gracias a los principios básicos utilizados para la construcción de árboles de decisión. Dentro de los principios tomados de los árboles de 
decisión para ser aplicados en el simulador se encuentran:

- Se analizan todas las posibles soluciones

- Permite analizar las probabilidades y resultados

Con un análisis tipo grafos de probabilidades se obtienen las rutas más relevantes, llegando así a los diez escenarios de mayor probabilidad de ocurrencia. Finalmente, el estudiante selecciona de los diez escenarios, el escenario meta. Si bien, sobre el escenario meta se define la planeación estrategia de la compañía, se debe tener en cuenta para los diez escenarios más probables es recomendable hacer el análisis cualitativo y cuantitativo de riesgos para definir los planes de acción a seguir en el caso que existan desvíos con respecto al escenario meta.

\section{Desarrollo de la experiencia de aprendizaje}

El modelo de análisis que se utiliza en la simulación es creación del autor, como ya trató en este documento. Uno de los aportes más significativos es unificar diferentes metodologías de análisis estratégico y prospectivo para que el estudiante pueda llegar de una manera más sencilla a la identificación del escenario meta. Para este proyecto primero fueron identificadas las herramientas de análisis idóneas, después se depuraron y se estructuró la metodología, para ser llevada a un software. Lo que significa que la herramienta tecnológica se puso al servicio de un desarrollo metodológico que funciona sin un simulador, porque el estudiante podría aplicarlo en otros ambientes de aprendizaje no mediados con tecnología.

Con el equipo de coatuores se trabajó la implementación de la metodología como una estrategia de aprendizaje a través del aprovechamiento de las TIC. Se estructuró la estrategia pedagógica y la implementación de la metodología en una herramienta de software, para ello se contó con un equipo de pedagogos, diseñadores e ingenieros que trabajaron de acuerdo con los requerimientos académicos.

El estudiante para simular debe elegir treinta tendencias que considere serán claves en el futuro de la compañía y que corresponden a variables internas o externas. Cada variable debe ser clasificada de acuerdo con los cuadrantes del análisis DOFA (debilidad, oportunidad, fortaleza, amenaza). Adicionalmente debe determinar el impacto de la variable en una escala que va de 0 a 3 , donde 0 es un impacto nulo y 3 es un impacto alto. De las treinta variables el simulador elige las de mayor impacto, y el estudiante debe seleccionar las diez principales. 
De esta manera, el proceso de simulación le exige al estudiante un alto nivel de análisis porque si llega a evaluar inadecuadamente sus variables afectarán los resultados de la simulación.

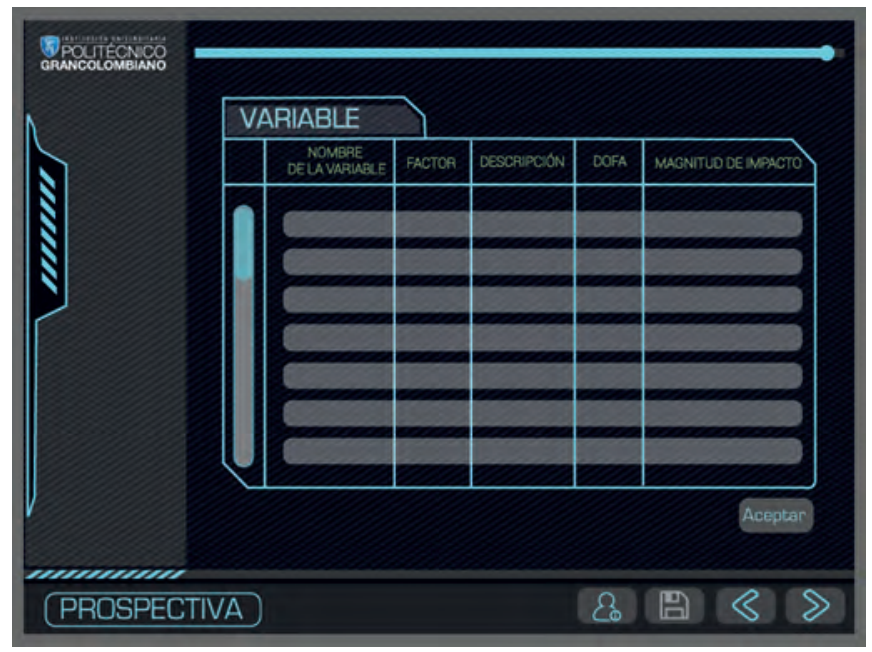

Figura 4. Escenario donde el estudiante ingresa la información de las variables en el simulador.

Fuente: Politécnico Grancolombiano.

Cuando elige las diez variables el simulador le genera la matriz de impactos cruzados, allí evaluará los niveles de dependencia e influencia de cada variable con respecto a las demás. El estudiante califica la relación entre variables siendo 0 Magnitud nula y 3 Magnitud alta.

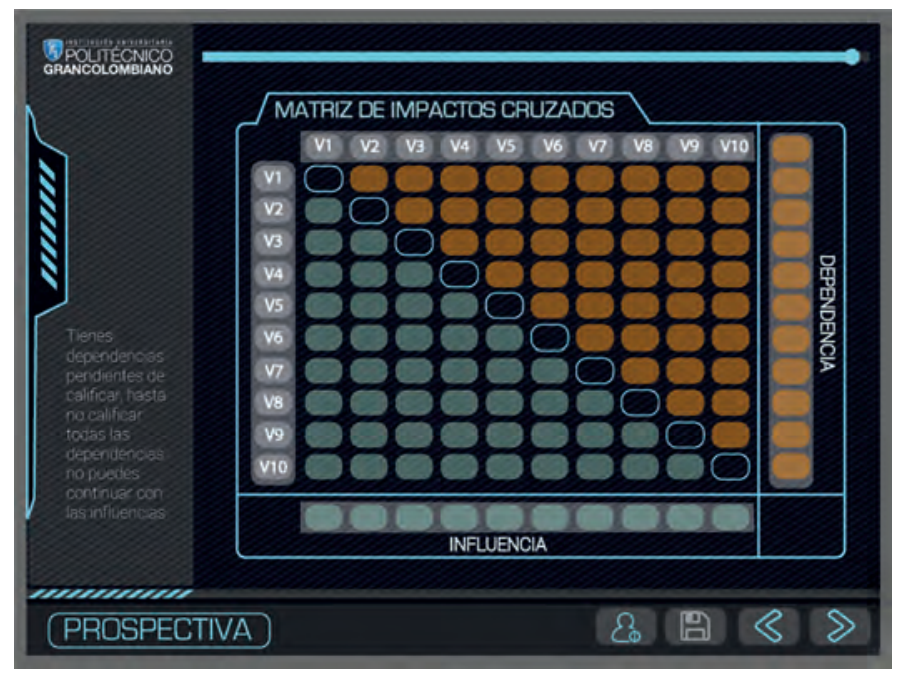

Figura 5. Escenario de la Matriz de Impactos Cruzados.

Fuente: Politécnico Grancolombiano. 
Con estos datos se genera el gráfico MIC MAC, el cual ubica todas las variables dentro de los cuadrantes del gráfico. De este gráfico el software selecciona las variables ubicadas en la zona de poder y en la zona de conflicto. Las variables que quedan fuera de la zona de poder y conflicto son descartadas para el análisis posterior, lo que significa depuración de variables para la creación de los escenarios.

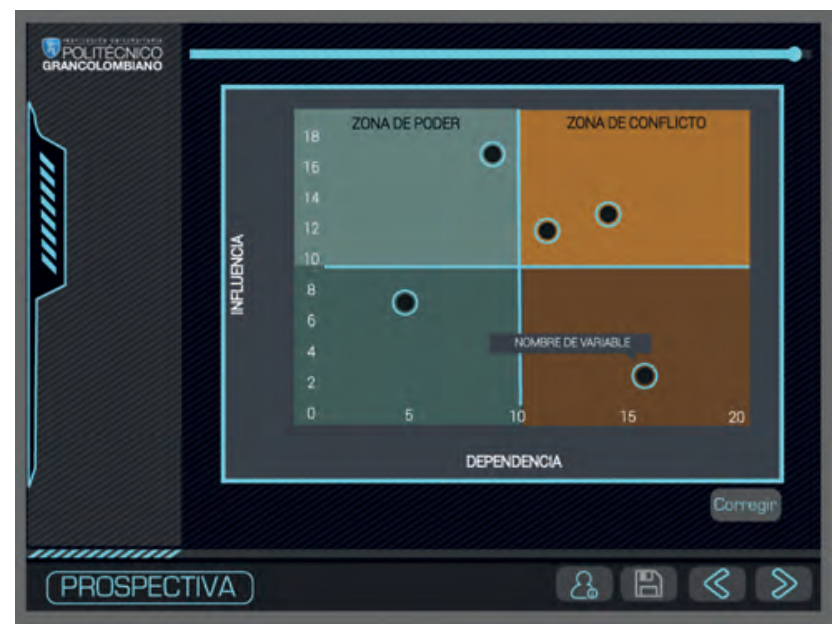

Figura 6. Escenario del gráfico MIC MAC.

Fuente: Politécnico Grancolombiano.

Con estas variables seleccionadas el estudiante procede a realizar las hipótesis para cada variable teniendo en cuenta los casos en los cuales existe alta dependencia entre variables.

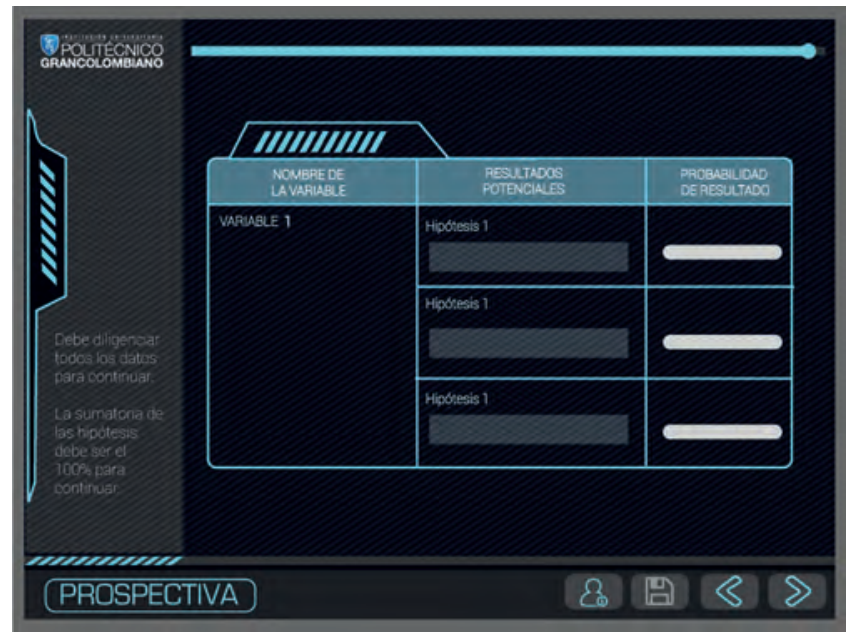

Figura 7. Escenario de la matriz de hipótesis.

Fuente: Politécnico Grancolombiano. 
A estas hipótesis el estudiante les asigna una probabilidad de ocurrencia de tal forma que el sistema hace una programación de grafos para elegir las rutas más probables y de esta manera arma los diez escenarios de mayor probabilidad de ocurrencia, los cuales se le muestran al estudiante y debe elegir el escenario meta.

El simulador le provee al estudiante los insumos para que con el escenario meta diseñe la planeación estratégica de la compañía. De aquí en adelante depende de un trabajo colaborativo donde el equipo redacta y construye el plan estratégico de la compañía para los próximos diez años, y finalmente, entrega al tutor un documento con los análisis realizados.

\section{Resultados obtenidos}

Los resultados del proyecto son el simulador individual y grupal y el espacio para el tutor. En el espacio individual el estudiante puede practicar las veces que desee con el fin de que adopte la metodología. En el espacio grupal, el equipo debe definir las variables y las hipótesis de manera mancomunada. El espacio dentro del simulador para la revisión y retroalimentación del tutor es el lugar donde revisa cada fase de la simulación hecha por el grupo y hace las retroalimentaciones necesarias. La simulación individual no es calificable, pero es requisito para acceder a la etapa grupal.

Además de la herramienta se elaboraron la guía metodológica para el estudiante y el instructivo de manejo de la herramienta. La primera orienta y da las instrucciones de cómo elaborar su proyecto y el proceso de simulación. El segundo es un instructivo de manejo de la herramienta. Los dos con el fin de constituir una experiencia completa de aprendizaje.

Se desarrolló una interface pensada en el vídeo juego que hace que la simulación sea muy dinámica, con unos personajes de instrucción e interacción para el estudiante que orienten los procesos y las tareas para el estudiante.

Para la institución quedan los desarrollos en html en código fuente que permite facilidades de programación para herramientas futuras. Todos los proyectos son construidos desde la perspectiva lego, es decir, que pueden pertenecer a este módulo $u$ otros similares. La misma visión le permite que se desarrolle por partes lo que significa que este simulador puede ser en futuro una parte $o$ componente de uno más grande. 


\section{Conclusiones}

La primera conclusión tiene que ver con la inexistencia en el mercado de un software que a través de una metodología ordenada permita la identificación de escenarios futuros en un contexto empresarial aplicable a cualquier tipo de empresa u organización desarrollada en una interfaz amigable y pedagógica.

El simulador puede ser aplicado a cualquier tipo de empresa y en cualquier etapa de su ciclo de vida, lo que facilita el análisis estratégico empresarial de largo plazo. Además, este permite desarrollar una experiencia de aprendizaje teórico-práctico, donde el estudiante se enfoca su trabajo esencialmente en el análisis y en el pensamiento crítico.

El desarrollo del simulador de Prospectiva como herramienta pedagógica, es un punto de partida para mejorar la calidad académica de los trabajos realizados por los estudiantes, del mismo modo, al estar diseñado para aplicar en empresas reales es una herramienta que fortalece la relación con el medio externo mediante el desarrollo de la investigación aplicada a la solución de problemáticas empresariales reales.

\section{Bibliografía o referencias}

Aguado, L. (2005). Emoción, Afecto y Motivación. Madrid: Alianza Editorial.

Akella, D. (2010). Learning together: Kolb's experiential theory and its application. Journal of Management and Organization, 16(1), 100-112.

Arango Morales X. y Cuevas Pérez V. (2014) Método de análisis estructural: matriz de impactos cruzados multiplicación aplicada a una clasificación (micmac). Universidad Autónoma de Nuevo León. Recuperado de: http://eprints. uanl.mx/6167/1/24.\%20capitulo\%20Metodologia\%20-\%20MICMAC\%20 \%28Direcci\%C3\%B3n\%20del\%20libro\%20a\%20la\%20venta\%20ttpwww.tirant. commexlibro9788416062324\%23\%29.pdf

Baena Paz, Guillermina; (2009). Prospectiva por qué y para qué: la historia que muchos no quieren leer. Estudios Políticos, Mayo-Agosto, 109-127.

Dalkey y Olaf, $(1962,07)$. An experimental aplication of the dephi metod to use of experts. Rand Co. Obtenido 09, 2017, de https://www.rand.org/content/dam/rand/pubs/ research_memoranda/2009/RM727.1.pdf

Godet, M., Monti, R., Meunier, F., \& Roubelat, F. (2000). La caja de herramientas de la prospectiva estratégica. Laboratoire d'Investigation Prospective et Stratégique.

Larmer, J. (2015). Project-Based Learning vs. Problem-Based Learning vs. X-BL.

Recuperado de: http://www.edutopia.org/blog/pblvs-pbl-vs-xbl-john-larmer [Consultado 10/09/2017] 
Mojica, F. (1992) La prospectiva. Técnicas para visualizar el futuro. Legis editores; Bogotá, $340 \mathrm{p}$.

Mojica F. (2006) Concepto y aplicación de la prospectiva estratégica. Revista Med Volumen 14. P (122-131). Recuperado de: http://www.umng.edu.co/ documents/63968/77289/RMed2006art15.pdf

Moore, D. (2013). For interns, experience isn't always the best teacher. The Chronicle of Higher Education. Recuperado de: http://chronicle.com/article/For-InternsExperience-Isnt/143073/ [Consultado 10/09/2017]

Moursund, D. (1999). Project-based learning using information technology. Eugene, OR, EUA: International Society for Technology in Education.

Quiroga D. (2008) Metodología para hacer prospectiva empresarial en la sociedad de la información y el conocimiento. Revista Economía y Administración № 70. Universidad Autónoma de Occidente; Cali.

Ryan, (2017, 01). Strategic Risk A cornerstone of risk transformation. Deloitte and Touche. Obtenido 09, 2017, de https://www2.deloitte.com/global/en/pages/risk/articles/ implementing-risk-transformation-in-organizations.html

Tziralis G. y Tatsiopoulos I. (2012) Prediction markets: an extended literature review. The University of Buckingham Press. Recuperado de: http://dx.doi.org/10.5750/jpm. v1i1.421

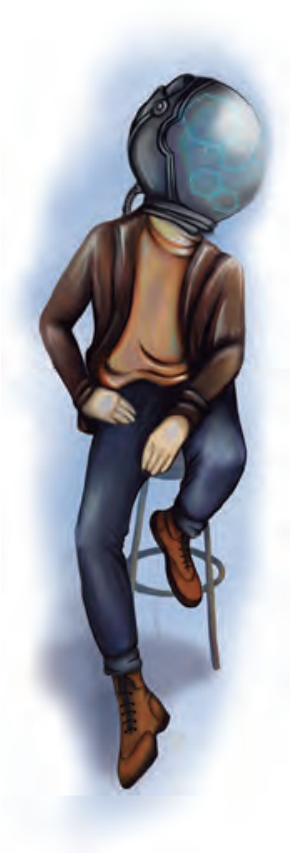

